\title{
Evaluation of the HB\&L system for the culture of prosthetic and osteoarticular origin samples
}

\author{
Agostina Ronca', Sabrina Brenci', Giuliana Carrega², Gianni Riccio², Luisa Santoriello' \\ I Unità Dipartimentale Semplice di Microbiologia-ospedale Santa Corona - Asl 2-Pietra ligure (SV) \\ 2 Unità Complessa di Malattie Infettive-Ospedale Santa Corona - As/2 Pietra Ligure (SV)
}

Key words: Prosthetic infection, osteoarticular infection, culture in liquid broth

Valutazione del sistema HB\&L per la coltura dei prelievi di origine protesica ed osteoarticolare

\section{SUMMARY}

Prosthetic and osteoarticular infections represent a complex condition to diagnose and resolve. In both cases, the eradication of microorganisms is difficult because of anatomical and physiological characteristics of the site of infection (bone). The best strategy for an effective pharmacological treatment is based on an early diagnosis confirmed by microbiological testing of bone, periprosthetic tissue or removed prostheses, to support clinicians to undertake prolonged targeted therapy. The purpose of this paper is to assess the clinical correlation between the results of the cultures performed with automated HB\&L (ALIFAX) system compared to the result of traditional methods.

HB\&L is a system to perform bacterial cultures, susceptibility and direct P.A.R. tests on biological materials based on detection of bacteria and fungi by laser light-scattering kinetics in liquid culture medium at $37^{\circ} \mathrm{C}$. Samples get to laboratory are inoculated in rich broth and placed in the incubator. The next day are processed accordin to the following protocol: $500 \mathrm{ml}$ of the broth are transferred in the vial of the instrument and $200 \mathrm{ml}$ of supplement DEB for fastidious organisms are added. The cultures are incubated for 360 minutes in the same time PAR test (antimicrobial residual power) is determined.

In the first half of 2009418 samples collected from prostheses and osteoarticular infections from 118 patients were analyzed. The tests showed 304 negative and II4 positive samples (27.30\%) from which were isolated Gram negative (I7.5\%) and Gram positive (82.5\%) bacteria. Gram positive included $38.3 \%$ S. aureus, $33 \%$ coagulase negative Staphylococci and $26.6 \%$ Enterococci.

Preliminary data obtained by the HB\&L system for the culture of tissue sample, as well as the improvement of surgical techniques have led to a significant increase in correlation with the clinical data compared to traditional microbiological analysis.

Le infezioni della protesi rappresentano una patologia complessa difficile da diagnosticare e da risolvere, una tra le complicanze più gravi e frequenti della chirurgia protesica.

I fallimenti asettici di un impianto sono frequenti, ma i quadri più gravi e le forme acute sono rappresentate dalle infezioni batteriche. Nel caso delle infezioni tipica è la difficoltà di eradicare l'agente infettante a causa delle caratteristiche anatomiche e fisiologiche della sede d'infezione (osso) e della presenza del corpo estraneo rappresentato dalla protesi (6).

Tale complessità impone spesso trattamenti chirurgici e farmacologici assai impegnativi e prolungati. Appare evidente quindi come sia assolutamente essenziale, prima di intraprendere qualsiasi trattamento, fare il massimo sforzo per ottenere una diagnosi microbiologica quanto più possibile accurata.

Lo scopo di questo lavoro è stato dunque quello di valutare la correlazione clinica tra i risultati delle colture eseguite con sistema automatizzato HB\&L (ALIFAX) in confronto alla metodica tradizionale.

Sono stati analizzati prelievi operatori di origine periprotesica ed osteoarticolare e sono stati scartati dalle conclusioni dell'indagine tutti i ceppi ripetuti e provenienti dallo stesso paziente, collezionati durante periodi lunghi di ospedalizzazione.

HB\&L(ALIFAX) è un sistema semiautomatico per l'esecuzione di colture batteriche, P.A.R. test ed antibiogrammi diretti su materiali biologici basato sulla rilevazione cinetica tramite laser light-scattering su mezzo di coltura liquido, in agitazione, termostatato a $37^{\circ} \mathrm{C}$.

I prelievi operatori dopo arricchimento in terreno liquido per 18 -24 ore a $37{ }^{\circ} \mathrm{C}$ sono stati processati secondo il seguente protocollo: nel vial di coltura dello strumento si sono inoculati $500 \mu \mathrm{l}$ di brodo arricchito e $200 \mu \mathrm{l}$ di DEB (terreno supplementare per microrganismi esigenti), incubando per 360 minuti; contemporaneamente sui campioni si è determinato il potere antibiotico residuo (P.A.R. test).

Identificazioni e antibiogrammi sono stati eseguiti con metodo automatizzato Phoenix (BD) e sono stati utilizzati i seguenti ceppi di controllo: E.coli ATCC25922, P.aeruginosa ATCC27853,S.aureus ATCC29213, E.faecalis ATCC29212,

\section{S.pneumoniae ATCC49619.}

Nel primo semestre 2009 sono stati analizzati 418 prelievi operatori di origine protesica ed osteoarticolare, provenienti da 118 pazienti (secondo le procedure chirurgiche si prelevano più campioni per paziente in maniera da ridurre la percentuale dei falsi positivi). Dall'analisi si sono ottenuti: 304 campioni negativi e 114 campioni positivi da cui si sono isolati 22 ceppi di Gram negativi e 92 ceppi di Gram positivi (38.3\% S.aureus, 33\% Stafilococchi coagulasi negativi e $26.6 \%$ enterococchi, $2.1 \%$ altro).

Prendendo in considerazione il numero dei pazienti da cui provenivano i campioni e scartando dall'analisi tutti i ceppi ripetuti isolati da uno stesso paziente, si può descrivere la condizione di questo primo semestre nel seguente modo: dei 118 casi analizzati si sono ottenuti 47 casi con coltura positiva, 51 con coltura negativa e 20 con coltura negativa, ma P.A.R. test positivo. Clinicamente invece, sono stati considerati come infetti 81 pazienti e 37 come non infetti.

Per quanto riguarda invece l'eziologia delle infezioni, dalle 47 colture positive si sono isolati 34 ceppi di Gram positivi, 7 ceppi di Gram negativi e 1 ceppo di Mycobacterium tuberculosis.

L'infezione di 5 pazienti è risultata essere a carico di una flora mista.

I microorganismi maggiormente isolati sono stati gli Stafilococchi spp. (29 ceppi), a seguire possono essere citati E. faecalis (4 ceppi), E. coli (1 ceppi) Pseudomonas aeruginosa (3 ceppi), Proteus mirabilis (2 ceppi), Salmonella typhimurium (1 ceppo) e M. tuberculosis (1 ceppo).

Gli Stafilococchi isolati possono essere suddivisi, oltre che per specie, anche in base alla meticillino- resistenza in 10 ceppi di Stafilococchi coagulasi negativi (CoNS) meticillinoresistenti, 9 ceppi CoNS meticillino-sensibili, 8 ceppi di Staphylococcus aureus meticillino-sensibile e 3 ceppi resistenti al farmaco.

In prima analisi si può affermare che la concordanza del dato microbiologico con coltura positiva con quello clinico è stata del $58 \%$.

\section{Corresponding author: Ronca Agostina}

U.D.S. di Microbiologia - Ospedale Santa Corona -Asl 2

17027 Pietra Ligure (SV) - Via XXV Aprile 38 - Tel.019/6234377

E-mail:agoronca@yahoo.it 
Tabella I. Correlazione tra dato microbiologico e dato clinico.

Coltura positiva $58 \%$

Coltura negativa, ma P.A.R. test POSITIVO $24 \%$

Dati non correlati $18 \%$

Dalla rielaborazione dei dati ottenuti, inoltre, si evince che l'utilizzo del sistema HB\&L (ALIFAX) per l'esame colturale in terreno liquido dei prelievi operatori ha migliorato la sensibilità diagnostica dell'analisi microbiologica (58\% vs $56 \%$ del metodo tradizionale (1).

Prendendo poi in considerazione il P.A.R .test (24\%), la correlazione tra dato microbiologico e clinico passa al $82 \%$ dal momento che le 20 colture microbiologicamente negative, ma con P.A.R. test positivo, sono clinicamente significative.

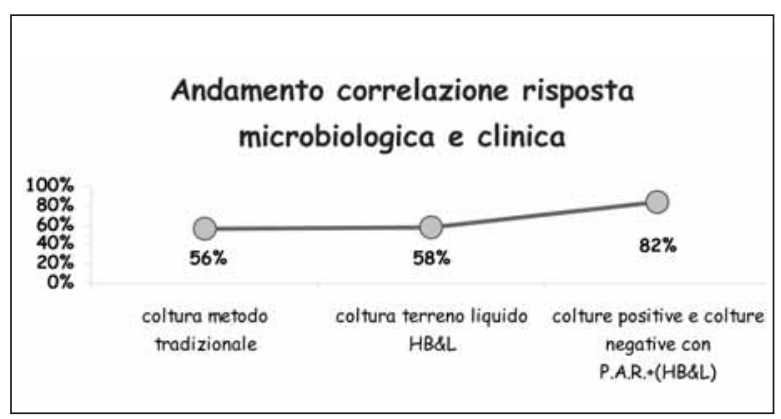

Figura I. Andamento correlazione tra dato microbiologico e clinica
Dalla letteratura è emerso inoltre che una migliore correlazione tra dato microbiologico e clinica ortopedica si ottiene dalla coltura diretta della protesi infetta asportata (65\%-94\%) (2). Tenendo conto che la patogenesi delle infezioni protesiche è la risultante tra l'interazione tra microrganismi (capacità di formare biofilm), impianto (caratteristiche chimico-fisiche di superficie) ed ospite (risposta infiammatoria)(6), tale coltura deve essere eseguita solo dopo trattamento con ultrasuoni della protesi stessa, allo scopo di rompere la matrice dello slime eventualmente prodotto dai batteri $(3,4,5,7)$.

Alla luce di questi dati nel nostro laboratorio è in fase di progettazione un nuovo protocollo diagnostico che preveda appunto la sonicazione della protesi asportate.

\section{BIBLIOGRAFIA}

1. Carrega G, Bartolacci V, Burastero G, et al. Aetiology of prosthetic joint infections in a terziari care centre in italy. Le infezioni in medicina, $\mathrm{n}$ 4,204-208,2008

2. Trampuz A et Al. Prosthetic joint infections: update in diagnosis and treatment; SWISS MED WKLY 2005; 135:243-25.

3. Trampuz A, Widmer AF. Infections associated with orthopedic implants. Curr. Opin. Infect. Dis. 19:349-356, 2006.

4. Trampuz A, Steckeldberg JM, et al. Advances in the laboratory diagnosis of prosthetic joint infection. Rew. Med. Microbiol 2003:14:1-14

5. Zimmerli W, Ochsner PE. Menagement of infection associated with prosthetic joints. Infection 2003;31:99-108.

6. Trampuz A., Kerryl E Piper et Al. Sonication of explanted prosthetic components in bags for diagnosi of prosthetic join infection is associated with risk of contamination. Journal of clinical microbiology, vol 44 n2 pp 628-631, 2006.

7. Trampuz A, Kerryl E Piper et Al. Sonication of removed hip and knee prostheses for diagnosis of infection. N Engl J Med 357:654-663, 2007. 